The Role of Disk-Halo Interaction in Galaxy Evolution: Outflow vs Infall? Editor

EAS Publications Series, Vol. ?, 2022

\title{
COSMIC RAYS IN THE DISK AND HALO OF GALAXIES
}

\author{
V.A.Dogiel ${ }^{1}$ and D. Breitschwerdt ${ }^{2}$
}

\begin{abstract}
We give a review of cosmic ray propagation models. It is shown that the development of the theory of cosmic ray origin leads inevitably to the conclusion that cosmic ray propagation in the Galaxy is determined by effective particle scattering, which is described by spatial diffusion. The Galactic Disk is surrounded by an extended halo, in which cosmic rays are confined before escaping into intergalactic space. For a long time cosmic ray convective outflow from the Galaxy (galactic wind) was believed to be insignificant. However, investigations of hydrodynamic stability and an analysis of ISM dynamics (including cosmic rays) showed that a galactic wind was emanating near the disk, and accelerating towards the halo, reaching its maximum velocity far away from the disk. Therefore convective cosmic ray transport should be important in galactic halos. Recent analysis of the gamma-ray emissivity in the Galactic disk of EGRET data, which showed that cosmic rays are more or less uniformly distributed in the radial direction of the disk, as well as the interpretation of soft X-ray emission in galactic halos, give convincing evidence of the existence of a galactic wind in star forming galaxies.
\end{abstract}

\section{Historical Review}

The story of cosmic rays (CRs) begins about 1900 when it was found that electroscopes (which measured the amount of ionization) discharged, even if they are kept in the dark well away from sources of natural radioactivity (for the history of CRs see the excellent monographs of Longair, 1992). In order to elucidate the role of the Earth, Hess and Kohlhörster carried out manned balloon flights. Especially successful was the flight of August 7, 1912 (birthday of CRs) when V. Hess reached an altitude of $5 \mathrm{~km}$. He found that the ionization rate there was several times higher than observed at sea level. Hess wrote:

\footnotetext{
${ }^{1}$ P.N. Lebedev Institute, Leninskii pr, 53, 119991 Moscow, Russia

2 Zentrum für Astronomie und Astrophysik, Technische Universität Berlin, Hardenbergstr. 36, D-10623 Berlin, Germany
}

(C) EDP Sciences 2022

DOI: (will be inserted later) 
2 The Role of Disk-Halo Interaction in Galaxy Evolution: Outflow vs Infall?

The results of the present observations seem to be mostly explained by the assumption that a radiation of very high penetrating power enters our atmosphere from above.

This radiation was named by Millikan in 1925 as cosmic radiation or cosmic rays (CRs).

The first characteristics of CRs were derived from measurements of their flux near Earth. It is quite amazing, how many global parameters of CR propagation in the Galaxy may be inferred from these "local" measurements. The most striking characteristic was that the spectrum of CRs in the energy range, covering six orders of magnitude, was found to be a power-law with a single index of $\gamma \simeq-2.7$, thereby strongly constraining the possible CR acceleration mechanisms. Another very interesting result obtained from measurements was that a group of light elements like $\mathrm{Li}, \mathrm{Be}, \mathrm{B}$ was enhanced by about five orders of magnitude in comparison with e.g. solar abundances. Although the fraction of these light elements in the total flux of CRs is negligibly small, we shall see how much they allow us to learn about CR propagation in the Galaxy.

The first question arising from the early measurements was: where are CRs produced, in our galaxy, or outside? In models of extragalactic CR origin, particles observed near Earth traveled from extragalactic sources to our Galaxy before reaching the solar system. Then, at least in the vicinity of the Galaxy (or, even, in the local group of galaxies) the density of CRs should be the same as near Earth, i.e. $w_{c r} \sim 10^{-12} \mathrm{erg} \mathrm{cm}^{-3}$. It is then easy to predict the expected flux of gamma-rays from $\pi^{\circ}$ decays from the nearest galaxies, i.e., the Small and Large magellanic Cloud (SMC, LMC), since the total mass of hydrogen there is known. In 1972 Ginzburg presented this criterion and estimated the flux from LMC for the extragalactic model, which was about $10^{-7} \mathrm{ph} \mathrm{cm}^{-2} \mathrm{~s}^{-1}$ for $E_{\gamma}>100 \mathrm{MeV}$. Only in 1993 an upper limit of the gamma-ray flux was obtained for this galaxy (Sreekumar et al., 1993), which was about $<0.5 \cdot 10^{-7} \mathrm{ph} \mathrm{cm}^{-2} \mathrm{~s}^{-1}$. This means that the CR density in the local extragalactic medium is nonuniform, and CRs (at least below $10^{15} \mathrm{eV}$ as it turns out) are produced in the Galaxy. As potential Galactic sources supernova (SN) explosions were assumed. This idea was suggested by Baade and Zwicky in 1934. Then Ginzburg and Syrovatskii (1964) substantiated it in their classical monograph "The origin of CRs".

\section{The Leaky Box Model}

The interpretation of observational data required a simple model of CR evolution, which was put forward and subsequently called leaky-box or thick target model (for a review see e.g. Cesarsky, 1980). In this model effects of particle spatial propagation were neglected, meaning that either the particle spatial distribution was uniform or the evolution of particle spectra was "local" when the spatial coordinates are parameters only. Particles were thought to be trapped within a box with semipermeable walls, at which they were reflected, but also having a finite probability to escape ("leak") into intergalactic space. In the stationary 
case the equation for the $\mathrm{CR}$ density $N$ with energies $E$ has a very simple form

$$
\frac{d}{d E}\left(\frac{d E}{d t} N\right)+\frac{N}{T}=Q(E) .
$$

Here $Q$ describes particle injection (source term), $d E / d t$ stands for energy losses, and $T$ is the characteristic time of catastrophic losses or particle escape.

These equations allowed to interpret the above-mentioned overabundance of the nuclei from the Li, Be, B group. The over-abundance of light elements, which are not produced in the interior of stars, was thought to be caused by fragmentation in the ISM by inelastic collisions of CR primaries with background particles. Then the "source term" for secondary nuclei of sort $i$ by primary nuclei of sort $j$ can be written as $Q_{i}(E)=\sum_{j} \bar{n}_{H} v \sigma_{i j} N_{j}$, where $n_{H}$ is the average density of the background gas, $v$ is the velocity of primary nuclei, and $\sigma$ is the cross-section of fragmentation. Since continuous energy losses are negligible for relativistic CRs the density ratio of secondary $N_{s}$ to primary nuclei $N_{p}$ is

$$
\frac{N_{s}}{N_{p r}} \simeq \bar{n}_{H} \sigma c T
$$

The value $x=\bar{\rho} c T$, where $\bar{\rho}=\bar{n}_{H} m_{p}, m_{p}$ is the proton rest mass, is denoted as the grammage. From the observed ratio $N_{s} / N_{p r}$ near Earth it was derived that $x \simeq 10 \mathrm{gr} \mathrm{cm}^{-2}$ (see, e.g. Ferrando et al., 1991).

With this value of $x$, and, what is quite noteworthy, from measurements of the CR flux near Earth, it is possible to derive the total luminosity of CRs in the Galaxy. It is $L_{c r}=W_{c r} / T$, where $W_{c r}$ is the total energy of CRs in the Galaxy, and $T$ is the average lifetime of CRs, both values being unknown. Let us multiply the numerator and the denominator by $\bar{n}_{H} m_{p} c$ (where $m_{p}$ is the proton rest mass), and write $W_{c r}$ as $\bar{w}_{c r} V$, where $w_{c r}$ is the average energy density of CRs in the Galaxy and $V$ is the volume of the Galaxy filled with CRs. Then the product $M_{H}=\bar{n}_{H} V m_{p}$ is the total mass of hydrogen in the Galaxy, which is known from radio measurements. Assuming that the density of CRs does not vary much over the volume of Galaxy, the total CR luminosity becomes $L_{c r}=$ $w_{c r} M_{H} c / x$. All values of this ratio are known from observations: $w_{c r} \sim 10^{-12}$ erg cm${ }^{-3}, M_{H} \simeq 10^{43} \mathrm{~g}$, and $x \simeq 10 \mathrm{gr} \mathrm{cm}^{-2}$. Then the CR luminosity is an astonishing $L_{c r}=3 \cdot 10^{40} \mathrm{erg} \mathrm{s}^{-1}$, amounting to about $3 \%$ of the total Galactic SN (hydrodynamic) energy release rate!

Potential sources of CRs in the Galaxy are observed to have the following luminosities: 1. SNe: $10^{42} \mathrm{erg} \mathrm{s}^{-1} ; 2$. neutron stars: $10^{41} \mathrm{erg} \mathrm{s}^{-1} ; 3$. stellar winds of O/B stars: $10^{41} \mathrm{erg} \mathrm{s}^{-1}$; 4 . flare stars: $3 \cdot 10^{40} \mathrm{erg} \mathrm{s}^{-1}$, i.e. only about several percents of the energy of SNe should be transformed into the flux of CRs in order to provide their luminosity in the Galaxy.

Some isotopes of the $\mathrm{Li}, \mathrm{Be}, \mathrm{B}$ group are radioactive. For instance the isotope ${ }^{10} \mathrm{Be}$ decays into ${ }^{10} \mathrm{~B}$ within the characteristic half life time $\tau_{r}=2.2 \cdot 10^{6}$ years. If the decay time is of the order of the CR lifetime $T$, then the latter can be estimated 
4 The Role of Disk-Halo Interaction in Galaxy Evolution: Outflow vs Infall?

from the density ratio of stable secondary nuclei $N^{s}$ to radioactive secondary nuclei $N^{r}$. Indeed, from the leaky-box model this ratio is

$$
\frac{N_{l}^{r}}{N_{i}^{s}}=\frac{1 / T_{l}}{1 / T_{l}+1 / \tau_{r}} \frac{\sum_{j} n_{H} v \sigma_{l j}^{r} N_{j}^{p}}{\sum_{j} n_{H} v \sigma_{i j}^{s} N_{j}^{p}} .
$$

From measurements it was derived that the value of CR lifetime calculated in the framework of the leaky-box model is $T_{l} \sim 2 \div 3 \cdot 10^{7}$ years (see e.g. Ahlen et al. 2000). Two very important conclusions follow from the values of $x$ and $T$ :

- In $10^{7}$ years CRs cover a distance $10^{25} \mathrm{~cm}$, or $3 \mathrm{Mpc}$. On the other hand, the thickness of the galactic disk is only about $300-500 \mathrm{pc}$ and its radius is about 10-15 kpc. Then, the trajectories of CRs in the Galaxy should be strongly tangled, i.e they perform a random walk with many scatterings off magnetic field irregularities. Their propagation can simply be described by a diffusion in coordinate space;

- If we estimate the average density of the gas from the values of $x$ and $T$ we obtain the value $\bar{n}_{H} \simeq 0.25 \mathrm{~cm}^{-3}$, which is almost four times smaller than the average gas density in he galactic disk. This means that CRs spend most of their lifetime outside the galactic disk, in the so-called galactic halo surrounding the disk where the gas density is small. Since the half thickness of the disk is about $250 \mathrm{pc}$, the thickness of the halo should be at least 1 kpc.

Thus, it was concluded that the description of CRs in the framework of the leakybox model is strongly restricted, and it was necessary to abandon the leaky-box model.

\section{The Diffusion Model}

The idea about particle scattering in the interstellar medium (ISM) by magnetic fields was suggested by Fermi (1949), being a necessary condition for stochastic acceleration of CRs in the ISM. In 1964 Parker investigated qualitatively the process of charged particle scattering by magnetic fluctuations. He analyzed particle propagation along the magnetic field lines whose equations was: $y^{*}=C_{1}+F(x)$, $z^{*}=C_{2}$, where $y^{*}$ and $z^{*}$ are coordinates of magnetic field lines and the constant $C_{1}$ and $C 2$ specify the line. Thus, we have a uniform magnetic field, $\mathbf{e}_{x} B_{0}$, in the $\mathrm{x}$-direction and a magnetic field irregularity in the y-direction, $\mathbf{b}_{y}=\mathbf{e}_{y} d F(x) / d x$. The equation for propagation of a particle with the charge $Z e$, the mass $M$ and the velocity $\mathbf{v}$ is

$$
\frac{d \mathbf{v}}{d t}=\frac{Z e}{M c} \nabla \times[\mathbf{v} \cdot \mathbf{B}]
$$


Below one should use magnitudes: $\mathbf{B}=\mathbf{B}_{0}+\mathbf{b}_{y}$, where $|\mathbf{b}| \ll\left|\mathbf{B}_{0}\right|$. Then the particle displacement in $\mathrm{y}$-direction is

$$
y=\Omega \int_{-\infty}^{t} d \tau F\left(v_{\|} \tau\right) \sin (\Omega \tau),
$$

where $\Omega=Z e B_{0} / M c$ and $v_{\|}$is the velocity component along $x$-direction. For the irregularity in the form $F(x)=a \exp \left(-x^{2} / b^{2}\right)$ a displacement of the particle pitch angle (angle between vectors of magnetic field and particle momentum) is derived from

$$
\frac{\triangle v_{\perp}}{v_{\|}}=\sqrt{\pi} \frac{a}{b} \frac{b^{2}}{R^{2}} \exp \left(-\frac{b^{2}}{4 R^{2}}\right)
$$

where $R=v / \Omega$ is the particle Larmor radius. One can see from this equation that interactions of particles with magnetic perturbations are resonant, and particles are scattered most efficiently by irregularities when the scale of magnetic field fluctuations is of the order of the particle Larmor radius, $a \sim R$.

We conclude from Eq. (3.3) that a single interaction of a particle with a resonant irregularity changes its pitch angle $\theta$ by $\triangle \theta \sim b / B_{0}$. Since particles are scattered by fluctuations randomly the total angle displacement after $N_{s c}$ interactions is proportional to $\sqrt{N_{s c}} b / B_{0}$, and therefore in order to be scattered through the angle $\pi$, we have the condition $\sqrt{N_{s c}} b / B_{0} \sim \pi$. The distance necessary to be scattered through $\pi$ is $\lambda_{s c} \sim N_{s c} R \sim\left(B_{0} / b\right)^{2} R$. Then we can introduce a diffusion coefficient for this random walk of a particle along magnetic field lines $D \sim \lambda_{s c} v$.

In 1965 Parker published a paper, in which he presented a phenomenological diffusion equation for CRs in interplanetary space. He concluded "that the feature of magnetic field, which determines the nature of the propagation of energetic particles is the presence of small-scale irregularities. The irregularities appear with dimensions comparable to the radius of gyration. The irregularities scatter, or reflect, the energetic particles back and forth along the line of force of the largescale field, so that there is no tendency for the particles to move systematically in either direction. Then the effect of magnetic irregularities is to cause the CR particles to random walk. The random walk of the CR particles is a Markov process, describable by a Fokker-Planck equation:"

$$
\frac{\partial N}{\partial t}+\frac{\partial}{\partial x_{j}}\left(N v_{j}\right)+\frac{\partial}{\partial E}\left(\frac{d E}{d t} N\right)-\frac{\partial}{\partial x_{j}}\left(K_{i j} \frac{\partial N}{\partial x_{i}}\right)=0 .
$$

A formal and correct derivation of the diffusion approximation for multiple scattering of charged particles in a random magnetic field was performed by Dolginov and Toptygin in 1966 (see translation of this paper Dolginov and Toptygin, 1967). Unfortunately, this analysis is almost unknown in the astrophysical community.

In the ISM magnetic irregularities are plasma waves whose magnetic field strength can be described as a spectrum

$$
\mathbf{b}(t, \mathbf{r})=\sum_{\alpha} \int d^{3} k \exp \left[-i \omega^{\alpha}(\mathbf{k}) t+i \mathbf{k} \cdot(\mathbf{r})\right] \mathbf{b}^{\alpha}(\mathbf{k}),
$$


where $\alpha$ denotes a certain species of plasma waves, which is determined from the corresponding dispersion relation $\omega=\omega^{\alpha}(\mathbf{k})$. This relation together with the condition of resonant particle-wave interaction, which in this case has the form

$$
\omega^{\alpha}(\mathbf{k})-k_{\|} v_{\|} \mp \omega_{H}=0
$$

determines the conditions of particle scattering in the ISM. Here $\omega_{H}=\Omega / \gamma$ is the relativistic gyrofrequency. The frequency of particle scattering (over pitch angles) is in this case

$$
\nu_{\mu}^{\alpha} \simeq 2 \pi^{2}\left|\omega_{H}\right| \frac{k_{r e s} W_{r e s}^{\alpha}\left(k_{r e s}\right)}{B_{0}^{2}}
$$

The value of $k_{\text {res }}$ is determined from the resonant condition (3.6), which for the case of MHD waves has the form $k_{\text {res }}=1 /(R|\mu|)$. Here $\mu$ is the cosine of particle pitch angle, and $W(k)=b^{2}(k) / 4 \pi$ is the wave energy density.

If scattering is so effective that the flux of propagating particles is izotropized then the Fokker-Plack equation can be simplified and reduced to the diffusion equation. The diffusion coefficient along the magnetic field line is calculated from the equation

$$
D=v^{2}\left[\frac{9}{2} \int_{0}^{1} d \mu\left(1-\mu^{2}\right) \nu_{\mu}\right]^{-1}
$$

This approximation can definitely be used for cosmic rays because their flux, as follows from observation, is highly isotropized, for details of the diffusion approximation, see Kulsrud and Pearce (1969) or the monograph by Berezinskii et al. (1990).

The major advantage of using the diffusion equation to describe particle propagation is the possibility to properly analyze the various cosmic ray components whose distribution in the Galaxy is strongly nonuniform. As an example, we mention the result of Prishchep and Ptuskin (1975) who showed that $T_{l}$ derived from the leaky-box model, Eq.(2.3), underestimated strongly the CR lifetime. What we infer from this model is a combination of the real CR lifetime $T_{D}$ (which in the framework of the diffusion model is $T_{D} \sim h^{2} / D$, where $h$ is a size of the halo) and the radioactive decay time $\tau_{r}, T_{l} \sim \sqrt{T_{D} \tau_{r}}$.

The diffusion approximation allowed to analyze the parameters of nonthermal emission generated by different CR components in different regions of the galactic disk and halo. In this way it was possible to estimate the size of the CR halo $h$. The diffusion model successfully described the measured parameters of the CR flux near Earth and the spectral characteristics of radio and gamma-ray emission (for details, see the monograph of Berezinskii et al. (1990), and for a similar, but more detailed, analysis based on the numerical program GALPROP of Strong and collaborators in the review of Strong et al., 2007)). However, this analysis rested on two phenomenological simplifications whose validity was not proven: a) it was supposed that all CRs independently of their energy and charge escape into the extragalactic space from the same surface of the halo, and, b) the effect 
of CR convective transport was ignored. Below we present arguments that these simplifications are in general not appropriate.

\section{Boundary Conditions on the Halo Surface}

In the monographs of Ginzburg and Syrovatskii (1964) and Berezinskii et al. (1990) (as well as in the GALPROP program) it was assumed that the boundary conditions for all components of CRs at the halo surface $\Sigma$ is $\left.N\right|_{\Sigma}=0$, i.e. all CR particles escape from the same surface. Dogiel et al. $(1993,1994)$ tried to derive these boundary conditions from kinetic equations. They analyzed the spherically symmetric case for the magnetic field, which is described by the equation:

$$
\mu v \frac{\partial f}{\partial r}+v \frac{1-\mu^{2}}{r} \frac{\partial f}{\partial \mu}=\frac{\partial}{\partial \mu}\left[\left(1-\mu^{2}\right) \nu(r, E, \mu) \frac{\partial f}{\partial \mu}\right]+Q(E) \delta(r) .
$$

By variable transformation this equation can be reduced to the case of particle propagation along a divergent magnetic flux tube.

The source of particles, $Q$, is assumed to be at the coordinate origin, and $\nu(r, E)$ is the scattering frequency. The problem was analyzed for an infinite space and it was supposed that the frequency of particle scattering decreases with the distance from the sources. Then the solution can be expanded in a Legendre polynomial series: $f(r, E, \mu)=f_{0}(r, E)+\delta^{-1} f_{1}(r, E, \mu)+\delta^{-2} f_{2}(r, E, \mu)+\ldots$, where $\delta=\nu / c(\partial f / \partial r)$. It was shown that for $\delta \gg 1$ the kinetic equation reduces to the diffusion equation with the coefficient $D \sim c^{2} / 3 \nu$. However, on the surface, where $\delta \sim 1$, scattering is not sufficient to isotropize the distribution function. For the frequency $\nu=\nu_{0}\left(E / E_{0}\right)^{-\alpha}\left(r / r_{0}\right)^{-b}$ this surface is located at:

$$
\bar{r}(E) \simeq r_{0}\left[\frac{\nu_{0}\left(E / E_{0}\right)^{-\alpha} r_{0}}{c}\right] .
$$

At distances larger than $\bar{r}(E)$ the solution of Eq. (4.1) is strongly anisotropic

$$
\begin{aligned}
& f(r, E, \mu)=\frac{C(E) \xi}{\xi+\tau(r, E)} \exp \left[-\frac{(1-\mu)(r / \bar{r})^{2}}{\xi+\tau(r, E)}\right], \\
& \tau(r, E)=\frac{4 \nu_{0}\left(E / E_{0}\right)^{-\alpha}(r / \bar{r})^{3-b}}{3-b},
\end{aligned}
$$

and the parameters $C$ and $\xi$ are determined from the conditions at $r=\bar{r}$.

The conclusion of this analysis is that CRs escape freely (i.e. run-away particles) from this surface along the magnetic field lines with their proper velocities.

Moreover, if magnetic fluctuations in the halo are excited by CRs due to the streaming instability whose increment is (Cesarsky 1980)

$$
\Gamma(k)=\frac{\Omega}{\gamma} \frac{N(>\bar{E})}{n}\left(\frac{\bar{V}}{V_{A}}-1\right),
$$

where $\bar{E}$ is derived from the resonant condition, $n$ is the density of the background plasma, $\bar{V}$ is the average velocity of the CR flux long the magnetic field lines, and 
$V_{A}=B_{0} / \sqrt{4 \pi n m_{p}}$ is the Alfvén velocity), the position of the run-away surface is determined by a single parameter, i.e. by the power of CR sources: the higher the power, the further away from the sources is the run-away (halo) surface. It was also shown that the halo size is a function of CR energy, and thus the halo radius decreases when the particle energy increases.

\section{Cosmic Ray Propagation: The Effect of Convection ("Galactic Wind")}

For a long time the effect of convective transport of CRs (the second term in Eq.(3.4) ) was not considered as significant, though in a number of papers the effect of convection on the CR spectrum was analyzed (see Bulanov et al. 1972, Jokipii 1976, Dogiel et al. 1980 etc.). The reasons were the following: a) the diffusion model was generally successful in accounting for most CR observations, both direct observations of particles near Earth as well as indirect observations like the diffuse radio-synchrotron and gamma-ray emission of our Galaxy; b) investigations did not imply that convective transport could be completely excluded, but wind velocities in the Galactic disk were only of the order of $10 \mathrm{~km} \mathrm{~s}^{-1}$ or less (for comparison the escape velocity from the Galaxy is about $500 \mathrm{~km} \mathrm{~s}^{-1}$ ).

However, a series of publications drew more attention to the process of CR transport (galactic wind). The first one was a paper by Wentzel (1974), who analyzed the so-called effect of self-confinement of CRs. CRs excite turbulence by the instability (4.4) to the level when the streaming velocity of CRs equals the velocity of self-excited waves. CRs are frozen into these fluctuations and move with their velocity.

In Breitschwerdt et al. (1991, 1993), and later in Zirakashvili et al. (1996), the system of cosmic ray hydrodynamic equations was analyzed, describing the balance of mass, momentum and energy together with the energy balance of CRs and hydromagnetic fluctuations in the Galaxy. They showed that, indeed, the convective velocity is small near the galactic plane, but the wind is accelerated by gaseous, CRs and MHD wave pressures, and as a result the wind velocity reaches the value of several hundred $\mathrm{km} \mathrm{s}^{-1}$ far away from the Galactic plane. Numerical calculations showed that the wind velocity increased almost linearly with distance close to the Galactic plane.

If CRs couple to the plasma via waves, a galactic wind develops, and CRs are picked up at the height $z_{c} \sim D / V$ by the wind and carried away from the galaxy. Hence the convective boundary is of the same importance as a run-away boundary for free particle escape. In general, $z_{c}$ is also a function of energy.

However, attempts to analyze the CR chemical composition in the framework of an accelerating wind resulted in an unexpected conclusion. According to the wind model, the convective boundary $z_{c}$ should be fairly close to the Galactic plane, i.e. $z_{c} \leq 1 \mathrm{kpc}$ (see Bloemen et al. 1990). The problem was that in this case, it was impossible to reconcile the chemical composition data with the almost unform CR distribution in the galactic plane, inferred from EGRET observations (see Strong and Mattox, 1996). The reason is the following: the spatial distribution of CR sources (SN remnants, superbubbles) is strongly nonuniform in the disk. It has a 
peak at a Galactocentric radius between 4 and $6 \mathrm{kpc}$ (coinciding with the observed molecular ring of the Galaxy), and drops off exponentially at larger distances. If the convective boundary is very close to the Galactic plane, the CR density is determined by the local source density, and therefore their distribution in the disk should follow closely the source distribution.

The only viable solution in the framework of the diffusion model is an efficient mixing of particles from different sources, implying a huge CR diffusion halo in order to wipe out the observed $\gamma$-ray gradient. However, even in the most favorable case of an extremely extended halo, the standard model is unable to remove the signature of the source distribution (see Dogiel and Uryson 1988, Bloemen et al. 1993).

One way to escape this dilemma is to assume that some of the observational data are not significant, like e.g. the SN distribution derived from radio observations because of absorption (Strong et al. 2000). However, Dragicevich et al. (1999) and Sasaki et al. (2004), analyzing SN distributions in external galaxies (where the effect of absorption is negligible), found similar radial SNR distributions as in our Galaxy. Therefore, the alternative is to conclude that it is time to abandon the standard diffusion model.

Paradoxically, as it may seem, the reason for a uniform CR distribution may indeed be a convective boundary close to the disk, if one accepts the possibility that CRs are also transported into the halo locally as a result of a chimney type flow. In, e.g., Bloemen et al. (1993) it was assumed that the location of the boundary is independent of Galactocentric radius. However, from a physical point of view, it is more reasonable to assume that the boundary is determined by the pressure driving the outflow, i.e., it the higher the pressure the closer it is to the disk. This was the ansatz of the model of Breitschwerdt et al. (2002), who were able to show, that a higher SN rate led to a rapid transport of CRs out of the Galaxy and thus reduced the $\gamma$-ray flux locally. In their comprehensive analysis of 1-D, 2-D and 3-D convection models they were able to explain an overall shallow $\gamma$-ray gradient as a result of a quasi-uniform distribution of CRs in the galactic disk.

The main effect can be described in the following way. The pressure of CRs is proportional to the density of CR sources, $P_{c r} \propto Q$. Since the convection velocity $V$ is proportional to the pressure, $V \propto P_{c r} \propto Q$, CRs leave the Galactic disk faster from a region of higher source density, and therefore the lifetime of CRs there is shorter than in other parts of the disk. The density of CRs in the disk can be estimated as $N_{c r}(r) \propto Q(r) / T_{c r}(r)$. We see that a high rate of CR production by source at any galactocentric radius is compensated by faster CR remove from this region. This effect cannot be obtained in the framework of a standard diffusion model. In this respect the "CR gradient in the disk" is an important piece of evidence in favor of a galactic wind in the Galaxy. Recently, Everett et al. (2008) have presented further support for a CR driven galactic wind by showing that soft $\mathrm{X}$-ray observations in a certain Galactic region can be better explained by a wind than by a hydrostatic halo model.

In a companion paper (Breitschwerdt et al. 2008, these proceedings) we will 
10 The Role of Disk-Halo Interaction in Galaxy Evolution: Outflow vs Infall?

discuss the properties of galactic winds, and show that soft X-ray halos in external galaxies can be naturally explained in a self-consistent galactic wind model with non-equilibrium ionization structure.

VAD is partly supported by the RFBR grant 08-02-00170-a, the NSC-RFBR Joint Research Project No 95WFA0700088 and by the grant of a President of the Russian Federation "Scientific School of Academician V.L.Ginzburg". DB thanks the organizer for some financial support.

\section{References}

Ahlen, S.P., Greene, N.R., Loomba, D. et al. 2000, ApJ, 534, 757

Baade, W., and Zwicky, F. 1934, Phys.Rev., 46, 76

Berezinsky, V.S., Bulanov, S.V., Dogiel, V.A., Ginzburg, V.L., Ptuskin, V.S., 1990, Astrophysics of Cosmic Rays, (ed. V.L.Ginzburg), North Holland.

Bloemen, J.B.G.M., Dogiel, V.A., Dorman, V.L., Ptuskin, V.S., 1993, A\&A, 267, 372

Breitschwerdt, D., McKenzie, J.F., Völk, H.J., 1991, A\&A, 245, 79

Breitschwerdt, D., McKenzie, J.F., Völk, H.J., 1993, A\&A, 269, 54

Breitschwerdt, D., Dogiel, V.A., and Völk, H.J. 2002, A\&A, 382, 730

Bulanov, S.V., Dogiel, V.A., Syrovatskii, S.I. 1972, Cosmic Research (in Russian: Kosmich. Issled.), 10, 653

Cesarsky, C.J. 1980, ARAA, 18, 289

Dogiel, V.A., Kovalenko, V.M., and Prishchep, V.L. Sov. Astron. Lett., 6, 366

Dogiel, V.A., and Uryson, A.V. 1988, A\&A, 197, 335

Dogiel, V.A., Gurevich, A.V., and Zybin, K.P. 1993, A\&A, 268, 356

Dogiel, V.A., Gurevich, A.V., and Zybin, K. P. 1994, A\&A, 281, 937

Dolginov, A.Z., and Toptygin, I.N. 1967, Soviet Physics JETP, 24, 1195

Dragicevich, P.M., Blair, D.G., Burman, R.R., 1999, MNRAS 302, 693

Everett, J.E., Zweibel, E.G., Benjamin, R.A., McCammon, D., Rocks, L., Gallagher, J.S., 2008, ApJ 674, 258

Fermi, E. 1949, Phys. Rev., 75, 1169

Ferrando, P., Lal, N., McDonald, F.B., and Webber, W.R. 1991, A\&A, 247, 163

Ginzburg, V.L. 1972, Nature, 239, 8

Ginzburg, V.L., and Syrovatskii, S.I. 1964, The Origin of Cosmic Rays, Pergamon Press

Jokipii, J.R. 1976, ApJ, 208, 900

Kulsrud, R., and Pearce, W.P. 1969, ApJ, 156, 445

Longair, M.S. 1992, High energy astrophysics. Vol.1: Particles, photons and their detection, Cambridge University Press

Parker, E.N. 1964, J. Geophys. Res., 69, 1755

Parker, E.N. 1965, Planetary and Space Science, 13, 9

Prishchep, V.L., and Ptuskin, V.S. 1975, Ap\&SS, 32, 265

Sasaki, M., Breitschwerdt, D., and Supper, R. 2004, Ap\&SS, 289, 283

Sreekumar, P., Bertsch, D.L., Dingus, B.L. et al. 1993, Phys. Rev. Lett., 70, 127

Strong, A.W., Mattox, J.R., 1996, A\&A 308, 21

Strong, A.W., Moskalenko, Igor V., and Ptuskin, V. S. 2007, Ann.Rev.Nucl.Part.Syst., 57,285 